University of Nebraska - Lincoln

DigitalCommons@University of Nebraska - Lincoln

Papers in Physical Properties

Chemical and Biomolecular Engineering

Research and Publications

$3-1-2003$

\title{
Effective Thermal Conductivity Estimates Of Particulate Mixtures
}

\author{
Christiaan Richter \\ Department of Chemical Engineering, University of Nebraska-Lincoln
}

Hendrik J. Viljoen

University of Nebraska - Lincoln, hviljoen1@unl.edu

N.F.J Van Rensburg

Department Mathematics, University of Pretoria, Pretoria, South Africa.

Follow this and additional works at: https://digitalcommons.unl.edu/chemengphysprop

Part of the Chemical Engineering Commons

Richter, Christiaan; Viljoen, Hendrik J.; and Rensburg, N.F.J Van, "Effective Thermal Conductivity Estimates Of Particulate Mixtures" (2003). Papers in Physical Properties. 1.

https://digitalcommons.unl.edu/chemengphysprop/1

This Article is brought to you for free and open access by the Chemical and Biomolecular Engineering Research and Publications at DigitalCommons@University of Nebraska - Lincoln. It has been accepted for inclusion in Papers in Physical Properties by an authorized administrator of DigitalCommons@University of Nebraska - Lincoln. 


\section{Effective Thermal Conductivity Estimates Of Particulate Mixtures}

Several theories have been advanced to estimate effective thermal conductivities of particulate mixtures, but most theories have focused on the dilute case. A method is proposed to estimate the effective thermal conductivity coefficient of mixtures of arbitrary concentration. Earlier the authors developed a theory to determine the expected contact area between different species. This theory is employed to determine the Kapitza resistance of the heterogeneous mixture and forms part of an overall theory to estimate the effective thermal conductivity. Results are compared with other theoretical estimates and with experiments.

\section{INTRODUCTION.}

A variety of methods have been proposed to estimate macroscopic physical properties of heterogeneous media. The analogies between five physical models: electrostatics, magnetostatics, thermal conduction, Euler fluids, and electrical conduction are widely appreciated. All the models describe a linear relationship between a driving force [negative gradient of a potential field] and a flux. The constant in the linear relation is expressed either as a conductance or as a resistance and in its more general form it constitutes a two- dimensional tensor. Most of the theories are based on the dilute [or linear] assumption. When a single particle is embedded in a matrix, it alters the potential field in the matrix. Invariably contributions of additional particles are considered additive and particle — particle interaction is neglected. These are also the fundamental premises of the Maxwell1 model later refined by Landauer,2 Kerner,3 and eventually Hashin4 to name only a few notable contributions. [The interested reader is referred to the book by Milton5 for a current review.] These earlier studies did not recognize the Kapitza effect, specifically present in heat transfer problems. A brief review of efforts to take into account the nonideal propagation of thermal phonons across a heteroboundary should be in order. Kapitza [cf. ter Haar] 6 first noticed discontinuous temperature fields at metal-liquid helium interfaces. Solid — liquid interfaces are expected to be more ideal than solid-solid interfaces, where the latter could include pores or trapped gas at the boundaries. Whenever the imperfection is of the order of a phonon wavelength or more, phonon transmission will be significantly impaired. The typical phonon frEquationuency is io" Hz and a typical phonon velocity for transversal modes would be 3000 mIs, thus the typical wavelength is on the order of atomic lattices and therefore quite susceptible to impedance mismatches. At the interface between two species the fluxes are continuous, but the temperatotare jumps according to the Kapitza resistance $R K$

$$
-k_{A} \nabla T_{A} \cdot n=-k_{B} \nabla T_{B} \cdot n=\frac{1}{R_{K}}\left(T_{A}-T_{B}\right) . \quad[1]
$$


Beneviste and Miloh' developed a general framework that incorporated thermal boundary resistance. In general this is primarily done by averaging all pertinent variables like heat flux and "intensity" over the composite medium viewed as a continuum consisting of a matrix with inclusions. This leads to a very general framework from which they were able to produce explicit expressions for the effective conductivity in the simplified case[s] of a composite consisting of a matrix with ellipsoidal inclusions embedded according to prescribed arrangements. One shortcoming was that it was still a "dilute theory" [i.e., neglecting interaction of inclusions], something Beneviste would soon remedy.8 Beneviste derived his model twice, first using the Kerner' and Hashin4 approach treating the matrix as an "effective medium" and then starting over employing the Mon-Tanaka theory. In both cases he got the same final result [for spherical inclusions] that we will henceforth report. For the sake of brevity, define $/ 3=a / k r n R K$, where $a$ denotes the radius of the particle inclusion. Also let $k$, and $k$, be the conductivity of the "matrix" or continuous phase and the "inclusions" or particulate phase, respectively. Then the effective thermal conductivity will be given by

$$
\frac{k_{\text {eff }}}{k_{m}}=\frac{2(1-\nu)+\beta\left[(1+2 \nu)+2 k_{m} / k_{P}(1-\nu)\right]}{2+\nu+\beta\left[(1-\nu)+k_{m} / k_{P}(2+\nu)\right]}[2]
$$

Hasselman and Johnson also corrected the Maxwell result' for Kapitza resistance and also arrived at Equation. [2]. Beneviste pointed out that Equation. [2] is valid for $v^{\prime} n / 6$, with s' being the volume fraction of the inclusion particles and the limit being the dense packing of spheres according to a simple cubic lattice [SCL]. Every et al used and further refined the effective medium approach. They presented Bruggeman's theory, corrected for Kapitra resistance, as a simple solution to the problem of high volume fraction of inclusions. As more inclusions are added to the matrix successively, the composite at the previous stage is considered as the matrix. The rate of change of effective conductivity with change in volume fraction is written in a differential form and the solution is

$$
(1-\nu)^{3}\left[\frac{k_{m}-k_{P}(1-\beta)}{k_{\mathrm{eff}}-k_{P}(1-\beta)}\right]^{3 \beta /(\beta-1)}=\left[\frac{k_{m}}{k_{\mathrm{eff}}}\right]^{(\beta+2) /(\beta-1)}
$$

$$
\frac{k_{\text {eff }}}{k_{m}}=\frac{\left[2(2-\nu)+2 k_{P} / k_{M}(1+\nu)\right]\left[4(1-\nu)+k_{P} / k_{M}(2+\nu)\right]}{\left[2(2+\nu)+2 k_{P} / k_{M}(1-\nu)\right]\left[(4-\nu)+2 k_{P} / k_{M}(1-\nu)\right]}
$$


Other improvements to the theory involve nonspherical inclusions. Special mention should be made of the important work of Nan]2 The T-matrix method, usually used for multiple scattering problems, has been adopted to estimate the effective thermal conductivity

$$
k_{\mathrm{eff}}=k_{\mathrm{hom}}+\langle T\rangle(I+\langle G T\rangle)^{-1}
$$

$$
\text { [5] }
$$

where the effective thermal conductivity has been split into a homogeneous part and a random fluctuating part, and G denotes a Green's function. The T matrix is simplified to keep it tractable by neglecting particle-particle interaction [a similar restriction as in the Maxwell model] and the model is expected to be inaccurate at $v$ irI6. The model reduces to Equation. [2] in the case of spherical inclusions.

It is fitting to conclude this brief overview with a quote from Hashin's paper: "It should be emphasized that $k e f f$ is not merely a function of $\mathrm{km}$. $k$, and $v$. It depends upon the entire statistics of the phase geometry. It is therefore a futile task to search for a theoretical expression for keff in terms of such simple geometrical information as phase volume fractions." In the following section we introduce a model that captures some of the statistics of the packing together with the material properties. But the model also has its limitations and this will be pointed out in the discussion.

\section{MATHEMATICAL MODEL}

Consider a mixture of species A and B, with volume fractions $\mathrm{p}$ and $\mathrm{I}-\mathrm{p}$. The discussion is limited to two species. A temperature gradient is applied across two parallel faces of the body and let it coincide with the $\mathrm{Z}$ axis of our coordinate system. No heat transfer occurs through the lateral surfaces and

$$
\begin{aligned}
& T=T_{H}, \text { at } \mathrm{z}=\mathrm{O} \\
& T=T c, \text { at } \mathrm{z}=\mathrm{H}
\end{aligned}
$$

As pointed out by Meredith and Tobias, 11 a serious limitation of Bruggeman's theory is that the suirounding matrix can be treated as a continuum only if successive inclusions are much larger than the ones added previously. It would thus only be valid for a large range of particle sizes. They corrected for this restriction by a stepwise addition of the particulate phase. Without consideration of Kapitza resistance, [3] their result [for spherical inclusions] is the goal is to derive an estimate for the effective thermal conductivity of the powder mixture, There are a number of auxiliary results that we will need in the derivation of the two estimates.

\section{A. Auxiliary results}

\section{The contact problem}

The issue of contact probability has been addressed and it is considered to be a solved problem. Richter and Viljoen'3 have developed a theory to determine the expectancy value AB of the surface contact area between two species A and B in the presence of a third species C. [In the original work three species have been considered, but we only need the result for two species in this work. The latter is merely a special 
case and the theory is presented in its original form.] It is necessary to know the surface areas of all species, but the species consist of particles with distributions in size and distributions in shape. In order to quantify these distributions, visualize an arbitrary particle from A. It is placed in the smallest possible rectangular rhombus with principal dimensions $\left[X_{A}{ }^{\prime} Y_{A}, Z_{A}\right]$. This approach provides us with three dimensions of the arbitrary particle and should give a reasonable representation, provided the particle is a convex body. Thus a probability density function $f\left[X_{A} Y_{A}, Z_{A}\right]$ [pdf] that describes the distribution of particles in three-

dimensional space contains information on shape, in contrast to one- dimensional distributions that are limited to information on size. The total surface area of each species can be calculated when the pdfs are known. All surfaces of the powder body are mapped onto each other and to determine Kapitza resistances, it is necessary to know the fraction of heterogeneously mapped surfaces.

When $C=0$, it is merely a special case of the three species problem. A brief description of this theory is in ordet Let the surface areas of $\mathrm{A}, \mathrm{B}$, and $\mathrm{C}$ be denoted by $\mathrm{S}_{\mathrm{A}}, \mathrm{S}_{\mathrm{B}}$ Sc Here the total area is $\Gamma=\mathrm{S}_{\mathrm{A}}+\mathrm{S}_{\mathrm{B}}+\mathrm{Sc}$. Introduce area fractions $\alpha=S_{A} / \Gamma, \beta=\mathrm{S}_{\mathrm{A}} / \Gamma$, and $\sigma=\mathrm{Sc} / \Gamma$ and define $\mathrm{a}=\min [\alpha, / 3]$ and $b=\max [\alpha, \beta]$

Now we are ready to construct a probability distribution for $\mathrm{A}-\mathrm{B}$ contacts based on the bijective rtiapping of discrete points on surfaces of species onto each other. The probability of $Z$ contacts between species $A$ and $\mathrm{B}$ in the presence of a third species $\mathrm{C}$ is given by the following expression [cf. Richter and Viljoen]

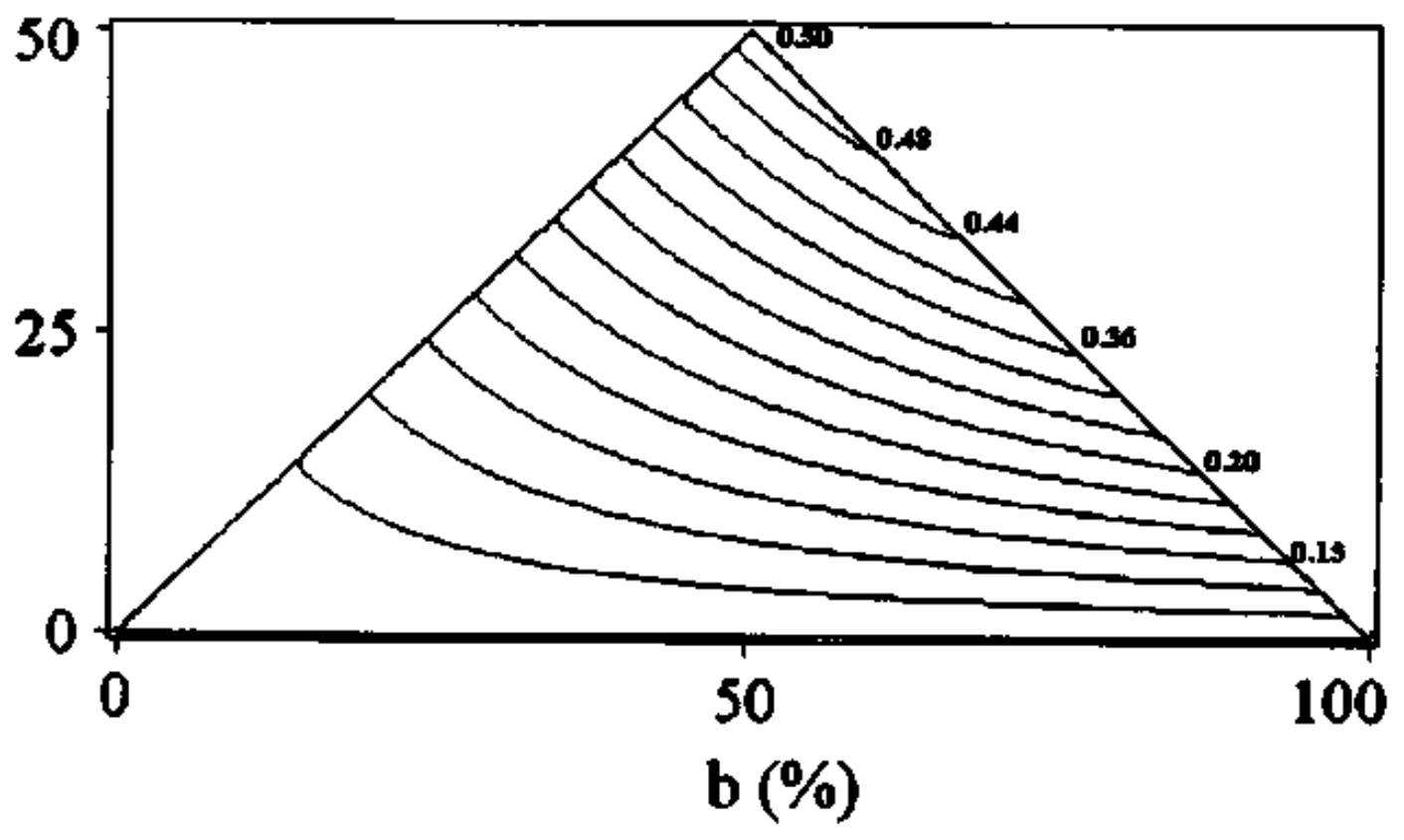

FIG. 1. Expectancy values. 


$$
\begin{gathered}
P(Z, a, b, \Gamma)=\frac{a \Gamma !}{Z !} \cdot \frac{b \Gamma !}{[b \Gamma-Z] !} \cdot \frac{\left(\frac{\Gamma}{2}\right) !}{\Gamma !} \cdot 2^{z} \cdot \sum_{k=0}^{\Delta} \frac{1}{k^{\prime} !} \\
\frac{1}{\left(\frac{a \Gamma-Z-k^{\prime}}{2}\right) !} \frac{c \Gamma !}{\left[c \Gamma-k^{\prime}\right]} \cdot \frac{\left((1-a) \Gamma-\left(Z+k^{\prime}\right)\right) !}{\left(\frac{(1-a) \Gamma-\left(Z+k^{\prime}\right)}{2}\right) !} \cdot 2^{k^{\prime}}
\end{gathered}
$$

[6]

where $\Delta \min [A-Z, C] 12, k=2 k$, when $\mathrm{Z}$ is even and $\Delta=\min [A Z-1, C-2] 12$ and $\mathrm{k}^{\prime}=2 \mathrm{k}+1$ when $Z$ is odd [consider 0 as even]. Let $c=\mathrm{I}-[a+b]$.

The first moment of this distribution function will give us the expectancy value we are interested in, namely the fraction of contact that is A on B.

Auxiliary result I:

$$
\Psi_{A B}(a, b)=\lim _{\Gamma \rightarrow \infty} \sum_{Z=0}^{a \Gamma} \frac{Z}{\Gamma / 2} P(Z, a, b, \Gamma)
$$

The expectancy function can be presented as a surface on the domain ax $b=[0,0.5] \times[0-\mathrm{I}]$. Considering any two of the three species, the minimum of the two will always he less than 0.5 and it is expedient in the calculation of $\boldsymbol{A B}$ to perform the calculations on this domain. A contour plot of the expectancy values corresponding to various A-B--C mixtures is shown in Fig. 1. Clearly these statistically predicted values are only valid when there is no significant bias to or against certain contacts due to differences in particle shape or cohesity. The numerical values of some contours are given to their right. 


\section{The progression problem}

Whether we view heat transfer either as phonon motion or the analogous electrical conduction problem, we are interested in the probable pathways that a phonon [or electron] could traverse. The progression problem deals with the probability that a phonon could progress from one layer to the next, but the pathway remains in only one type of species. The logic is that phonon travel will prefer the phase with higher conductivity. Without loss of generality, let that phase be A. The problem can be considered at different levels of complexity. First consider a $3 \times 3$ cube arrangement as shown in Fig. 2[a].

Starting at the center cube, the probability to go to the next level, by passing only through cubes of type A, can be calculated as follows. If the fraction of particles of type $A$ is $p$. there is a probability of $p$ that the cube directly above is of type A.

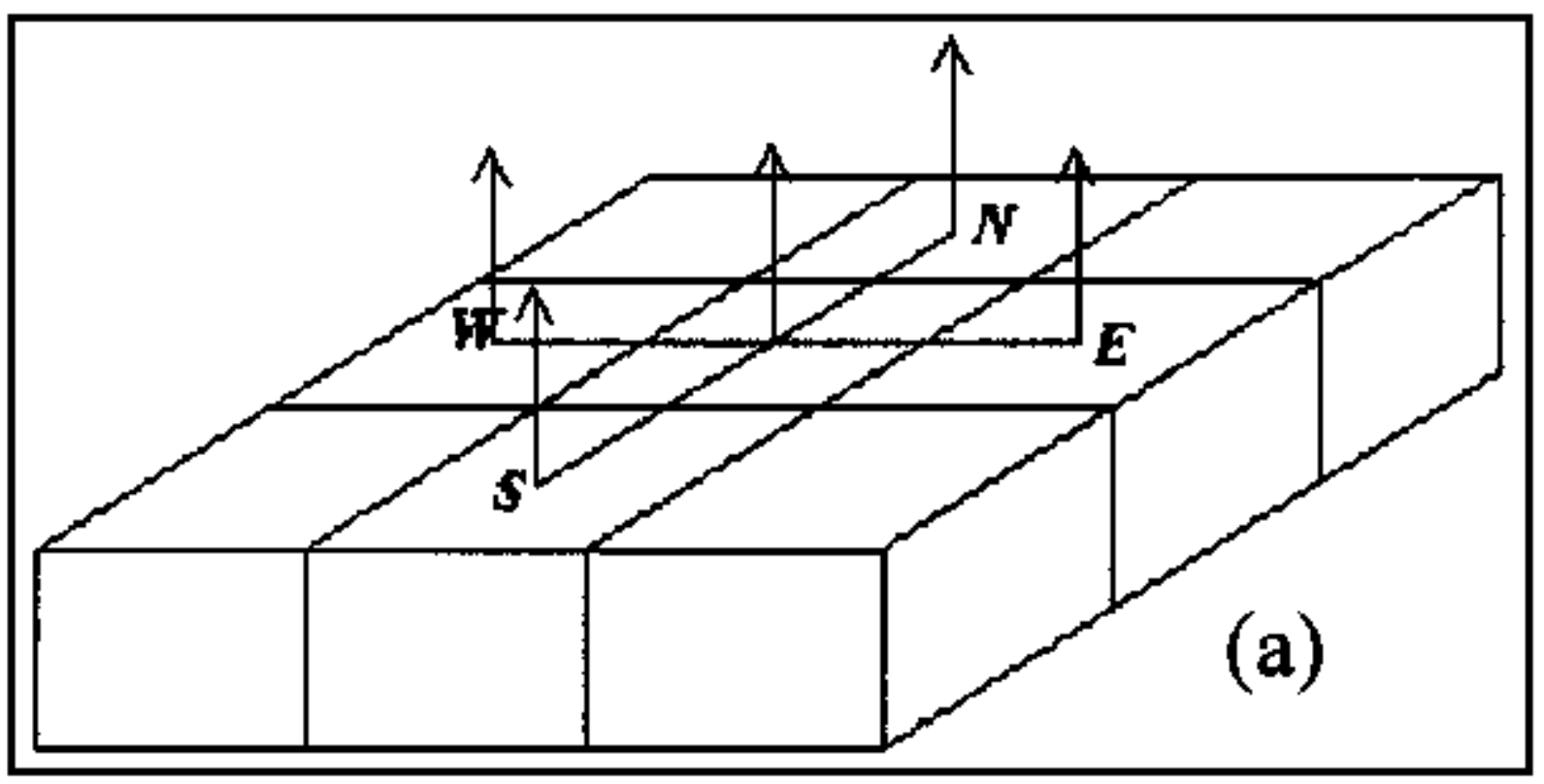




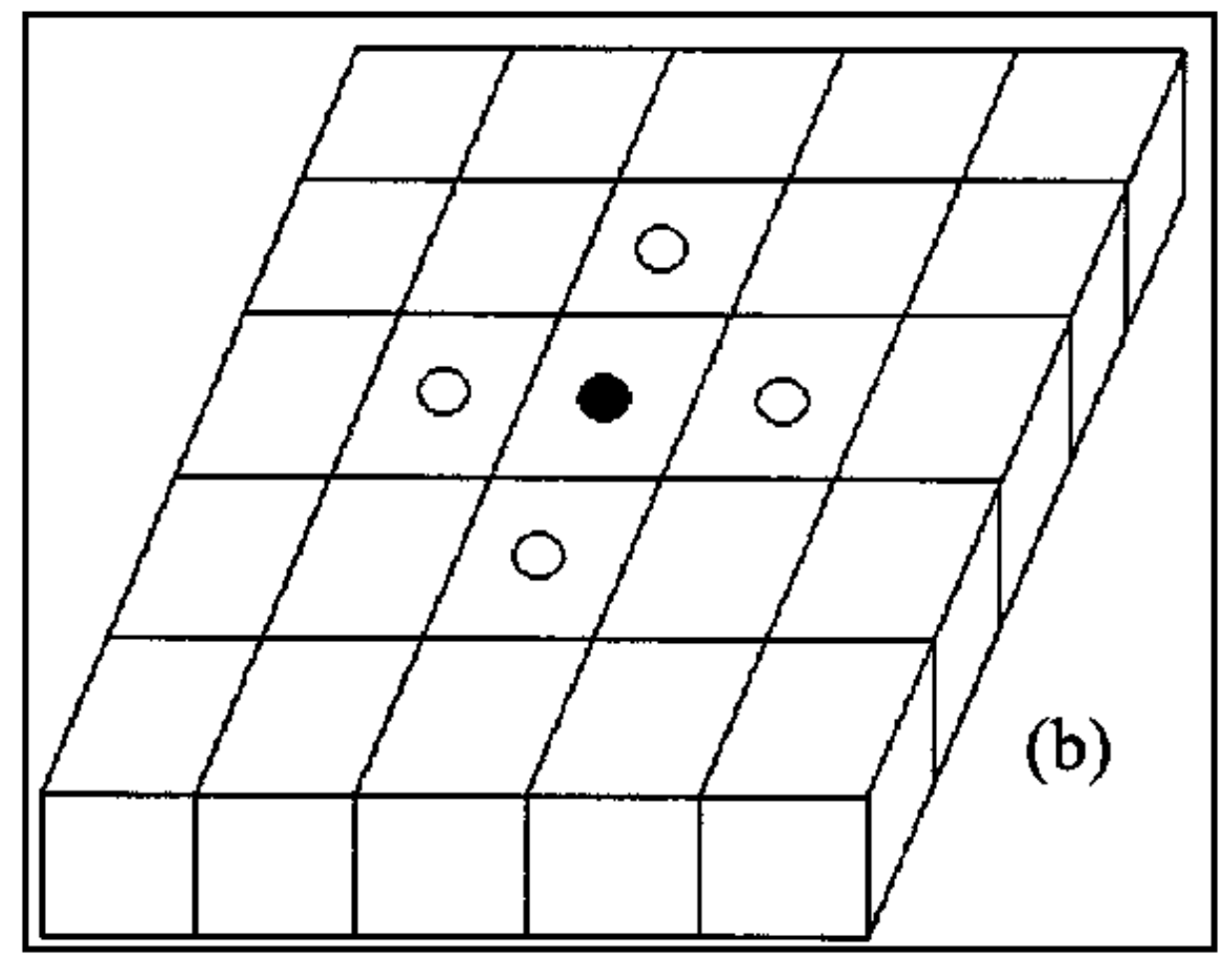

FIGURE. 2. [a] Progression through $3 \times 3$ array; [b] procession through a $5 \times 5$ array.

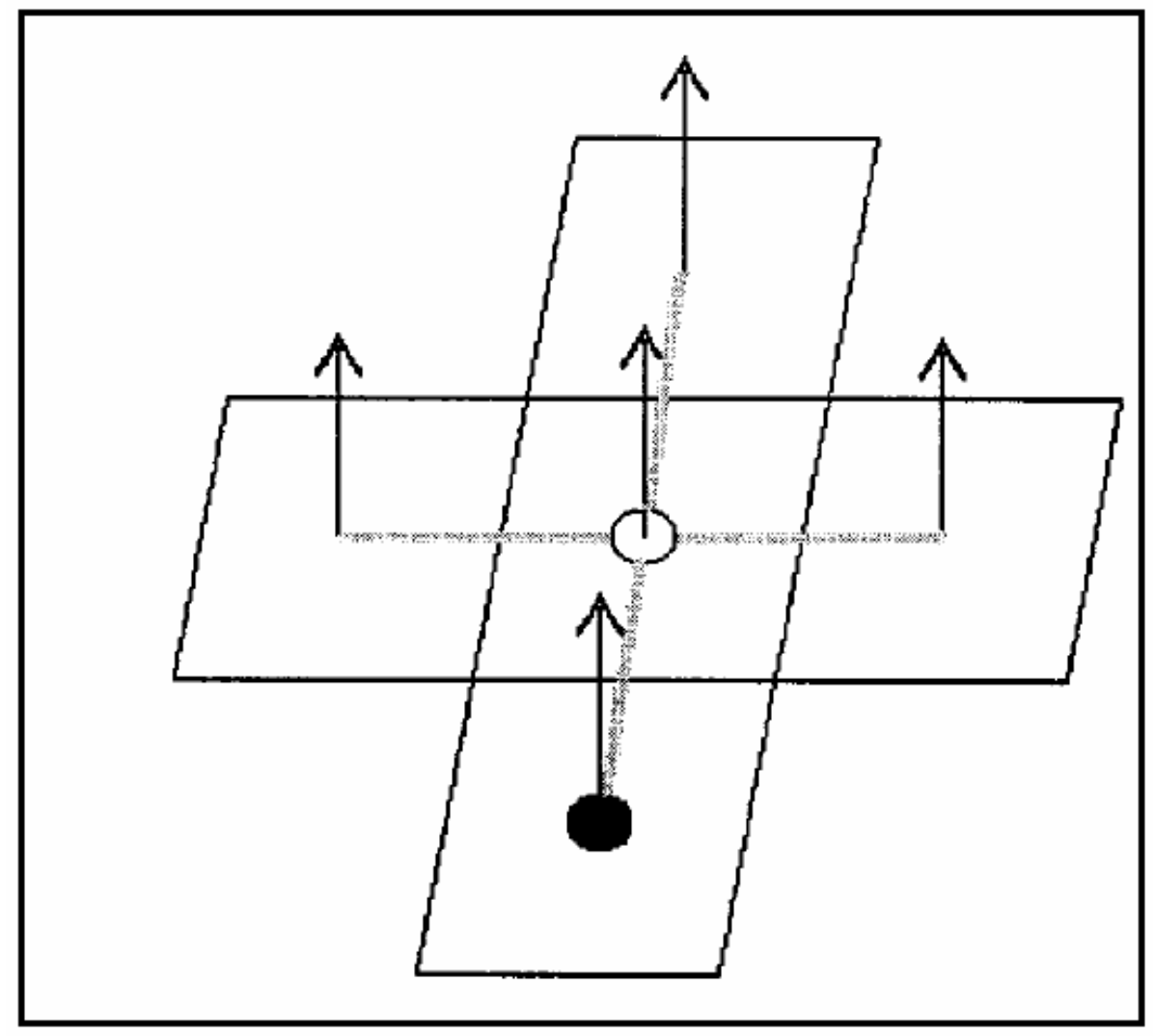


If this is not so, we could explore the four possibilities of moving north, south, east, or west on the cube arrangement and then up. [Diagonal motion is not considered.] The chance for success of one of these is $\mathrm{p} 2$. The chance for failure is thus $1-\mathrm{p} 2$ and the probability that all four possibilities will fail is [1 - p2]4 and the chance that one or more of these options may work is $1-[1-\mathrm{p} 2] 4$. We can then write the probability to move from one layer to the next according to this strategy

$$
P_{1}^{A}=\rho+[1-\rho]\left\{1-\left[1-1 \rho^{2}\right]^{4}\right\}
$$

[8]

In a $5 X 5$ cube arrangement on the same level, there is the additional possibility to make two lateral moves before moving up. This progression is schematically presented in Fig. 2[b]. The probability for this case gives

$$
P_{2}^{A}=P_{1}^{A}+P_{A}^{*}
$$

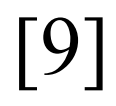

where $\boldsymbol{P}^{*}$ is given in the Appendix. In Fig. 2[b] the open and filled circles of the right hand side sketch must be transposed onto the open and filled circles of the left hand side to obtain all possible pathways.

In Fig. 3 the probabilities for all three approaches are shown and the convergence is obvious. The difference between curves $\boldsymbol{P}_{\boldsymbol{0}}{ }^{\boldsymbol{A}}\left[\boldsymbol{P}_{\boldsymbol{0}}{ }^{\boldsymbol{A}}=\boldsymbol{p}\right]$ and $\mathrm{P}_{1}^{\mathrm{A}}$ [Equation. [8]] is much larger than the difference between $\mathrm{P}_{1}{ }^{\mathrm{A}}$ and $\mathrm{P}_{2}{ }^{\mathrm{A}}$ consistent with the expectation that the probabilities of more complicated pathways would converge. There exists a competition between shorter pathways [of which there are few] and longer pathways [of which there are more]. When a preferred shorter pathway does not exist, the existence of one or more longer pathways becomes even less likely. This explains the convergencein Fig. 3. There is little gain in pursuing more complicated pathways.

Similar probabilities can be derived for passage through type B, merely exchanging $\mathrm{p}$ by $\mathrm{i}-\mathrm{p}$ in Equations. [8]- [9]. But it is important to distinguish between the higher conducting and poorer conducting species. Let A remain the higher conductinr species. The preferred nro2ression is directly up

through A with probability p, the next best choice is one of the pathways shown in Fig. 2[a]. As longer pathways through A are considered, the resistance increases. A point could be reached where the shorter pathway through B becomes favorable, despite its poorer conductivity. This argument can be formalized as follows. The number of steps that should be taken through A, before conduction through $\mathrm{B}$. must be considered, is; $N<k_{A} / k_{B}$, where species A could be either the particulate or matrix phases. This argument is not related to the earlier discussion on convergence of progressively longer pathways, which allows one to omit longer pathways for most calculations simply because their contribution becomes negligible. $N$ therefore formalizes a criterion for progression through $\mathrm{A} / \mathrm{B}$ follows; 


$$
\begin{gathered}
P_{A}=P_{n}^{A} \approx P_{j}^{A} \\
P_{B}=1-P_{A}=1-P_{n}^{A} \approx 1-P_{j}^{A}
\end{gathered}
$$

where $\mathrm{n}$ is the nearest integer less than $\boldsymbol{N}$ andj is any integer less than or Equationual to $n$ such that the approximation is good while $A$ and $\boldsymbol{F f i}$ are the overall probabilities of progressing through A or B. The limits are instructive. When $\boldsymbol{k} \boldsymbol{A}=\boldsymbol{k} \boldsymbol{8}$ then $n=0$, corresponding to a homogeneous material and when $\boldsymbol{k} \boldsymbol{B}-* \boldsymbol{0}$ [B is an ideal insulator] then $n-{ }^{\circ}$, that is progression must be through $\mathrm{A}$, and we arrive at an interesting percolation problem to be considered laten

The average distance that a phonon or electron travels when it progresses from one layer to the next is more than Ax and differs from one probability to the next. The average path length can be determined for these probabilities. Con side $\boldsymbol{P}$. It is written as three terms, consisting of the prob [8 ability to pass up directly, to make one lateral step and then pass up or to make two lateral steps and then pass up. The average path length, scaled by Ax, is easily obtained as etc.

$$
\begin{aligned}
& L_{1}^{A}=\left[\rho+2[1-\rho]\left\{1-\left[1-\rho^{2}\right]^{4}\right\}\right] / P_{1}^{A} \\
& L_{2}^{A}=\left\lfloor\rho+2[1-\rho]\left\{1-\left[1-\rho^{2}\right]^{4}\right\}+3 P_{A}^{*}\right] / P_{2}^{A}
\end{aligned}
$$

In Fig. 4 the average path lengths are shown for $\boldsymbol{P}$ and $\boldsymbol{F}$. It is interesting to note that the maximum path length occurs when the more conducting phase concentration lies between $30 \%$ and $40 \%$. The alternative pathways, associated with 1 and $\boldsymbol{P} \mathbf{1}$, extend the average path length considerably compared to $\boldsymbol{L I} \mathbf{1}$. A simple cubic lattice has been used to demonstrate the progression. The nodes are the positions of the particles' center of mass and the branches represent the shortest line connecting particles in contact. This is a similar configuration to the resistor network model that is used to study critical behavior. In the resistor networks the nodes are often referred to as sites and the connecting lines as branches [fuses]. Thus random branch removal from a network leads to a branch percolation model and random node removal is described by a site-percolation model. The latter is associated with the correlated removal of four branches for each site removal. The similarities with this model are obvious, although we are not concerned about the scaling behavior between driving force and flux. 


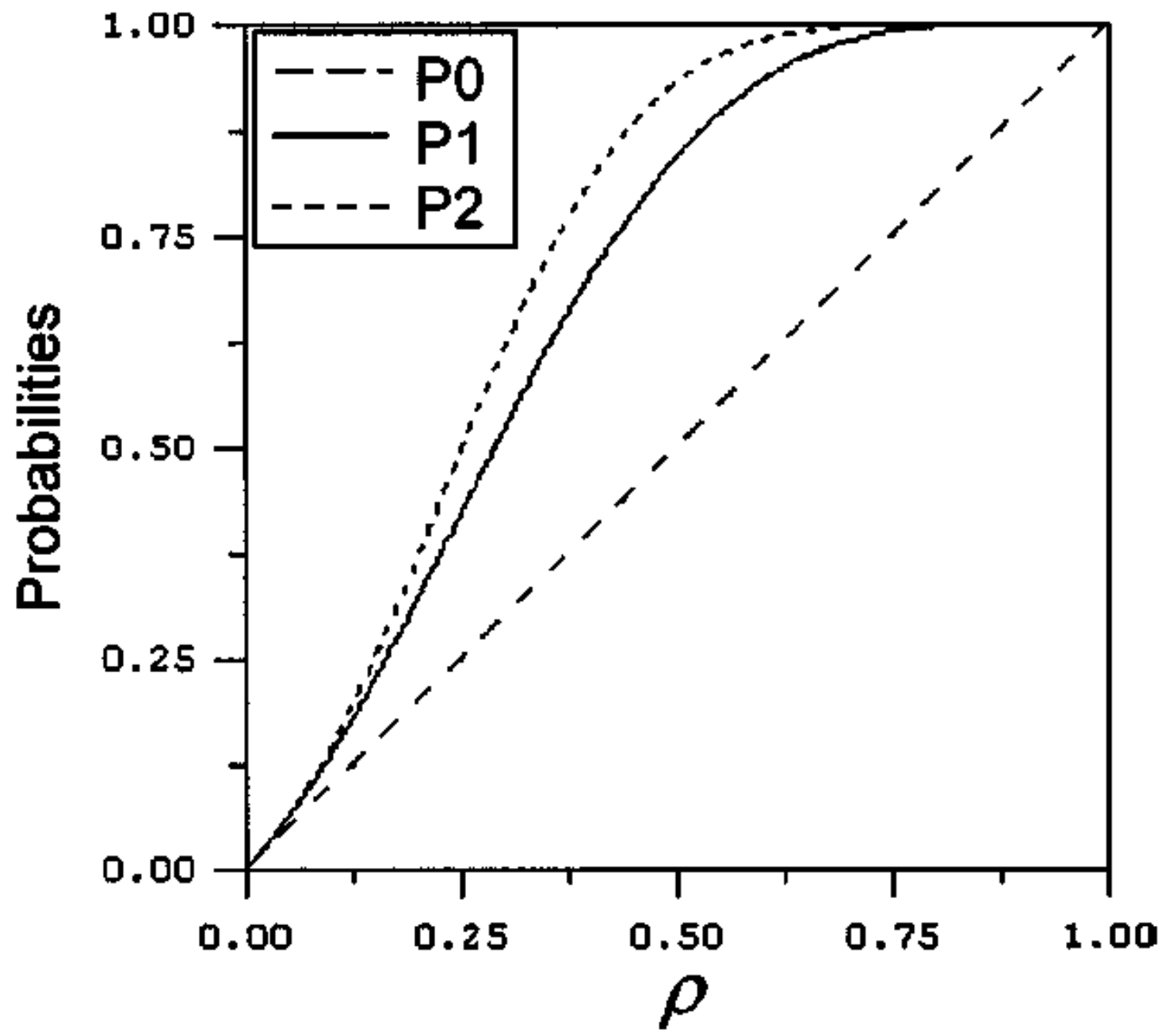

FIGURE. 3. $P 0 . P 1$, and $P 2$. 


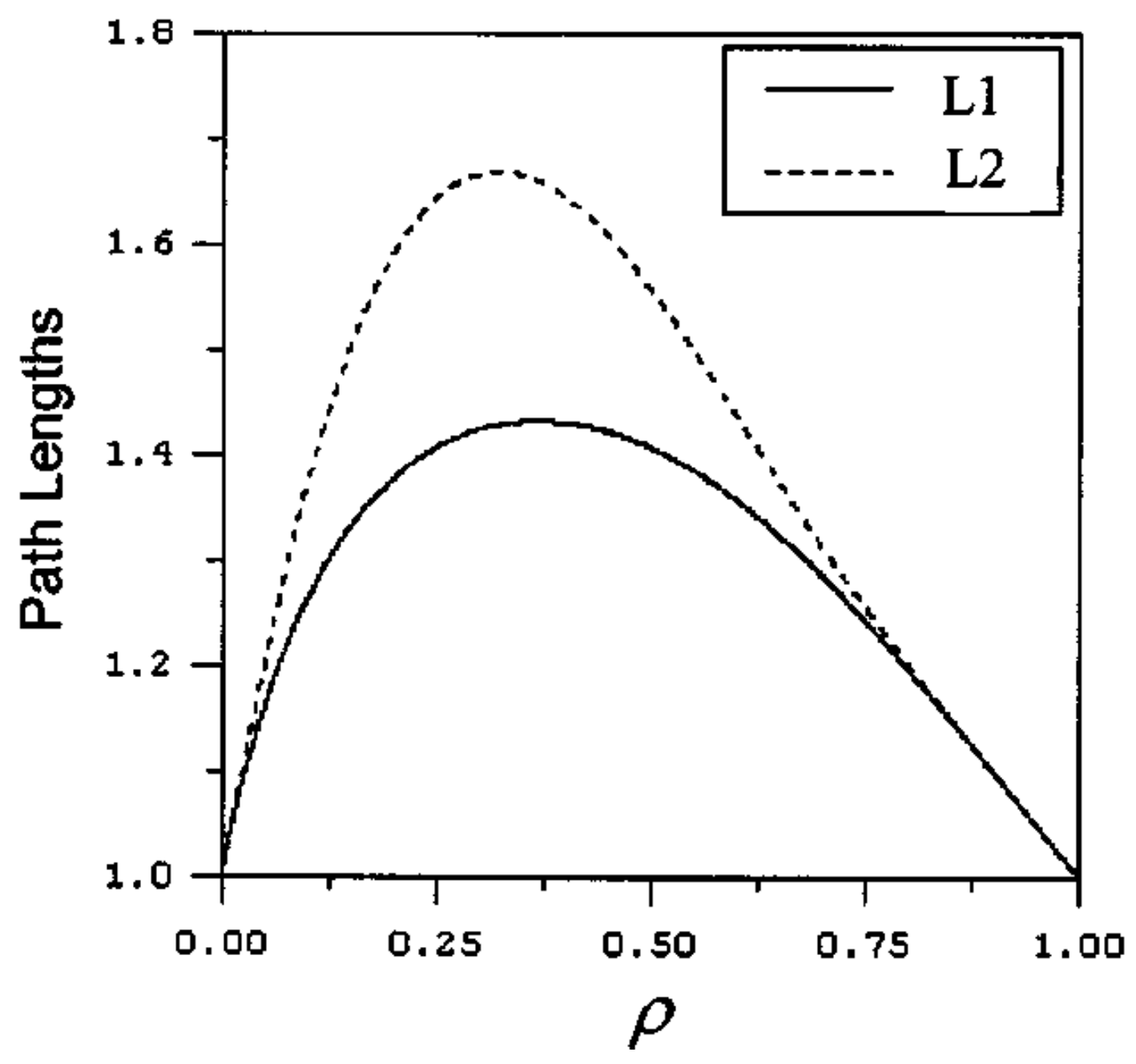

FIGURE. 4. Path lengths.

Two important points must be made: The simple cubic lattice does not imply that the particles are cubic. It depicts the avenge particle arrangement with coordination number 6 and the coordination is divided into I sup-coordination, 1 sub-coordination, and 4 in-plane contacts. Second, the probabilities for progression and average path lengths are independent of the formula still to be derived for effective thermal conductivity [ct Equation. [29]]. Thus we could use other contacting schemes, work out the probabilities $\boldsymbol{p}$, and path lengths $L^{\prime} 6$, and subsEquationuently use the results in Equation. [29].

Kurashige et aL14 did a numerical study of the effective thermal conductivity of a random sphere packing, under the influence of gravity. They found that the average coordination number was slightly larger than six and an investigation of the angular distributions of contacts showed a very isotropic azimuthal distribution, while the zenithal distribution was quite anisotropic. Let $P ø[\varnothing]$ and $P \theta[\theta]$ denote the distributions of coordination numbers in the zenithal and azimuthal planes. Thus we can determine the number of in-plane contacts and the above plane contacts 


$$
H=\int_{0}^{2 \pi} P_{\theta}(\theta) d \theta
$$

$$
V=\left[\int_{-\pi / 2}^{\pi / 2} P_{\phi}(\phi) d \phi-H\right] / 2
$$

A general progression probability can be derived along the same arguments as used for Equations. [8] - [9]. Thus the probability to propagate along species A, with density p, is Auxiliary result II:

$$
P_{A}=\left[1-(1-\rho)^{V}\right]+[1-\rho]^{V}\left\{1-\left[1-\rho+\rho(1-\rho)^{V}\right]^{H}\right\} \quad[16]
$$

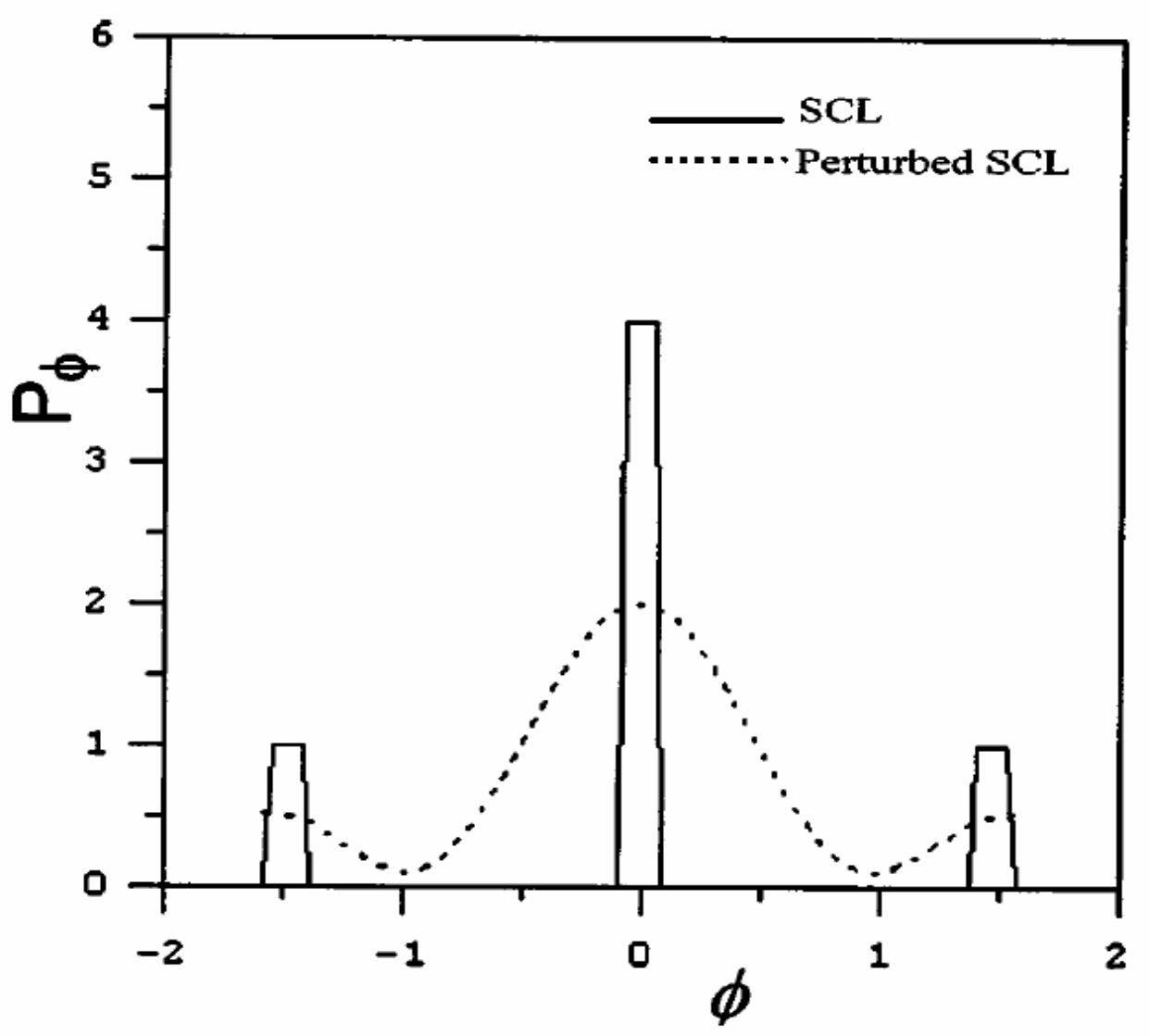

FIGURE. 5. Zenithal coordination distributions for: [I] a SCL and [2] a perturbed SC. 
Auxiliary result III:

$$
\begin{array}{r}
L_{A}=\left\{\left[1-(1-\rho)^{V}\right]+2[1-\rho]^{V} \times\left(1-\left[1-\rho+\rho(1-\rho)^{V}\right]^{H}\right)\right\} / P_{A} \\
{[17]}
\end{array}
$$

If the distributions are known, the horizontal and above- plane contacts $H$ and $V$ can be calculated. The probability to progress and the average path length follow from Equations. [6] - [7]. The conceptof in-plane contacts can be relaxed if it is recognized that particles need not lie in a perfect plane, permitting some randomness in the packing. For that purpose it is necessary to modify Equations. [14]- [15] slightly

$$
\begin{gathered}
H=\int_{0-\delta}^{0+\delta} d \phi \int_{0}^{2 \pi} P_{\theta}(\phi, \theta) d \theta \\
V=\left\{\int_{0}^{2 \pi} d \theta \int_{-\pi / 2}^{\pi / 2} P_{\phi}(\phi, \theta) d \phi-H\right\} / 2
\end{gathered}
$$

The out-of-plane angle $\mathrm{S}$ must be determined a priori and at this stage no better suggestion can be made than to experiment with that. In essence the somewhat arbitrary decision must be made when a neighboring particle lies in the same, but distorted, plane and when it actually lies in the next plane.

[15] In Fig. 5 the zenithal distribution of coordination is shown for a simple cubic lattice. [The peaks should actually be delta functions.] There is a single coordination below and above the horizontal plane at $4= \pm \mathrm{ir} / 2$ and a coordination of four at $4=0$. When the packing is perturbed, the coordination distribution looks like the second graph in Fig. 5. Starting with different ideal packings, e.g., hcp, bcc, etc., the distributions of the perturbed packings would be modified 16 accordingly. Kurashige et packed the domain randomly from the onset and observed a maximum in the coordination at $t h \pm$ "n-/4. It is conjectured that sufficient randomization regardless of the packing [but in the presence of gravity]. $Q A B=-p P$ [nniAx2]ATI[2Ax] should eventually lead to a distribution as found by Kurashige et al.

The azimuthal distributions for a SCL consists of delta functions of unit value at $\mathrm{fi}=0, \mathrm{n}-/ 2, \mathrm{r}, 3 \mathrm{n} 12$, thus there are a coordination of four in the plane. When this state is randomly perturbed, the distribution approaches Equationual probability to coordinate at any angle 0 in the horizontal plane. The necessary results are now in place that we can proceed with the derivation of the effective thermal conductivity, 


\section{b. Model of Effective Thermal Conductivity:}

Consider layers of particles A and B. Each particle has side length Ax and they are arranged as layers of $n X m$ and $<$ layers high. The volumetric fraction of $\mathrm{A}$ is $\mathrm{p}$. Species $\mathrm{A}$ and $\mathrm{B}$ have different thermal transport properties. If a homogeneous temperature is placed at the bottom and top of the stack insulated lateral walls!, we want to calculate the effective thermal conductivity of the stack. When only one layer is present, the fluxes through species A and B are

$$
\begin{aligned}
& Q_{A}=-k_{A} \rho\left(n m \Delta x^{2}\right) \Delta T / \Delta x \\
& Q_{B}=-k_{B}(1-\rho)\left(n m \Delta x^{2}\right) \Delta T / \Delta x
\end{aligned}
$$

The

effective thermal conductivity is

$$
k_{\mathrm{eff}}=\left[k_{A} \rho+k_{B}(1-\rho)\right]
$$

This is a simple mixing rule.

When two layers are condensed the following different types of fluxes exist.

$$
\begin{gathered}
Q_{A A}=-\rho P_{A}\left(n m \Delta x^{2}\right) \Delta T /(2 \Delta x) \times\left[\frac{1}{\left(1+L_{A}\right) / 2 k_{A}+1 /\left(2 \Delta x h_{A}\right)}\right] \\
Q_{B B}=-(1-\rho) P_{B}\left(n m \Delta x^{2}\right) \Delta T /(2 \Delta x) \times\left[\frac{1}{\left(1+L_{B}\right) / 2 k_{B}+1 /\left(2 \Delta x h_{B}\right)}\right] \\
Q_{A B}=-\rho P_{B}\left(n m \Delta x^{2}\right) \Delta T /(2 \Delta x) \times\left[\frac{1}{1 / 2 k_{A}+1 /\left(2 \Delta x h_{K}\right)+L_{B} / 2 k_{B}}\right] \\
{[25]}
\end{gathered}
$$

where we included the Kapitza conductances $h_{K}$ for A-B, $h_{A v}$ for A-A, and $h_{B}$ for B-B interfaces. Often the Kapitza resistances for at least some of these interfaces are negligible and can be omitted. The subscript of $Q$ identifies the path of each flux. By summing over all the fluxes the effective thermal conductivity is found to be 


$$
Q_{B A}=-(1-\rho) P_{A}\left(n m \Delta x^{2}\right) \Delta T /(2 \Delta x) \times\left[\frac{1}{1 / 2 k_{B}+1 /\left(2 \Delta x h_{K}\right)+L_{A} / 2 k_{A}}\right]
$$

$[26]$

$$
\begin{aligned}
k_{\mathrm{eff}}=2\left[\frac{\rho P_{A}}{\left(1+L_{A}\right) / k_{A}+1 /\left(\Delta x h_{A}\right)}+\frac{(1-\rho) P_{B}}{\left(1+L_{B}\right) / k_{B}+1 /\left(\Delta x h_{B}\right)}\right. \\
\left.\quad+\frac{(1-\rho) P_{A}}{1 / k_{B}+1 /\left(\Delta x h_{K}\right)+L_{A} / k_{A}}+\frac{\rho P_{B}}{1 / k_{A}+1 /\left(\Delta x h_{K}\right)+L_{B} / k_{B}}\right]
\end{aligned}
$$

$[27]$

The three-tier case becomes more involved since fluxes of the type $Q_{A A B}$ and $Q_{A B A}$ are not the same. Their general form is

$$
Q_{A I J}=-\rho\left(n m \Delta x^{2}\right) \Delta T /(3 \Delta x) \times\left[\frac{3 P_{A} P_{B}}{\left(1+L_{B}\right) / k_{B}+M /\left(\Delta x h_{B}\right)+N /\left(\Delta x h_{A}\right)+L_{A} / k_{A}}\right]
$$


where $M 51,2$ and $N 51,0$ for $I J 5 A B, B A$, respectively When $k 11$ layers are considered, the effective thermal conductivity can be written as and $a l=\min [l I k,[k-1] I k]$ and $b j=\max [l I I c,[k-l] I k]$. Also, $C=k ! /[k-I] t l !$ and $P[. l, k-l]$ is the expectancy value for the number of contacts between $I$ particles of type $B$ and $k$ -1 particles of type $A$.

$$
\begin{aligned}
k_{\mathrm{eff}}= & (1-\rho)(k+1)\left\{\left[\frac{P_{B}^{k}}{\left(1+k L_{B}\right) / k_{B}+k /\left(\Delta x h_{B}\right)}\right]\right. \\
& \left.+\sum_{l=1}^{k} \frac{\frac{C_{l}^{k} P_{B}^{k-l} P_{A}^{l}}{1+(k-l) L_{B}}}{k_{B}}+\frac{\Psi\left(a_{l}, b_{l}\right) k}{\Delta x h_{K}}+\frac{l-\Psi\left(a_{l}, b_{l}\right) k}{\Delta x h_{A}}+\frac{(k-l)-\Psi\left(a_{l}, b_{l}\right) k}{\Delta x h_{B}}+\frac{l L_{A}}{k_{A}}\right\}+\rho(k+1) \\
& \times\left\{\left[\frac{P_{A}^{k}}{\left(1+k L_{A}\right) / k_{A}+k /\left(\Delta x h_{A}\right)}\right]+\sum_{i=1}^{k} \frac{C_{l}^{k} P_{A}^{k-l} P_{B}^{l}}{\frac{1+(k-l) L_{A}}{k_{A}}+\frac{\Psi\left(a_{l}, b_{l}\right) k}{\Delta x h_{K}}+\frac{(k-l)-\Psi\left(a_{l}, b_{l}\right) k}{\Delta x h_{A}}+\frac{l-\Psi\left(a_{l}, b_{l}\right) k}{\Delta x h_{B}}+\frac{l L_{B}}{k_{B}}}\right\}
\end{aligned}
$$

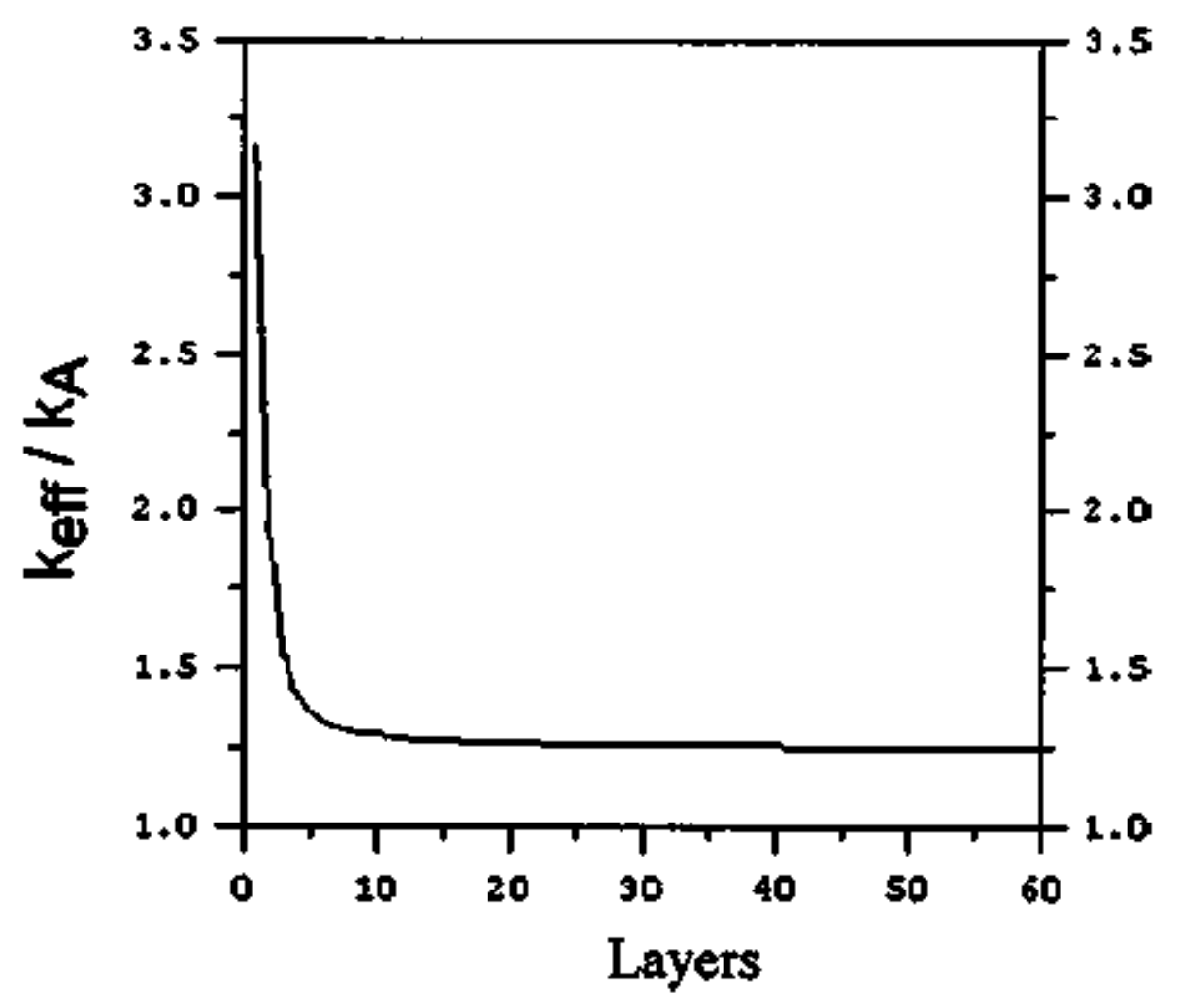


FIGURE. 6. Dependence of effective thermal conductivity on thickness.

A very important, albeit subtle point, is the usage of auxiliary result $I$ to find the number of hetero contacts and thus the Kapitza resistances.

An interesting consequence of this theory is that the effective thermal conductivity depends on the number of layers. It is obvious that the single and double layer estimates, Equations. [12] and [17], differ but one would also expect that the effective thermal conductivity would approach a constant asymptotic value for large layers. This turns out to be the case. While the probability for any specific path decreases with increasing layers, the number of paths increases as well and the total sum approaches a constant.

In Fig. 6 the effective thermal conductivity -is plotted as a function of the number of layers. The physical properties correspond to the values listed in Table I. The particle size is 4pm and p0.25.

At least ten layers are necessary for reasonable approximations. It can be concluded from this theory that the effective thermal conductivity of a thin composite depends on its thickness in the range of $1-10$ particle diameters.

\section{APPLICATION -}

The effective thennal conductivity, calculated from Equation. [29] is compared with the effective medium approximation of Nan,'2 [Equation. [2]18912 and Bruggeman's method [cf. Every'0 and Equation. [3]1. The results are compared to experimental results of Every et aL for $\mathrm{ZnS} /$ diamond.

\section{Table I}

\begin{tabular}{lll}
\hline \multicolumn{1}{c}{ Parameter } & \multicolumn{1}{c}{ Value } & \multicolumn{1}{c}{ Units } \\
\hline$h_{Z D}$ (Kapitza conductance) & $1.67 \times 10^{7}$ & $\mathrm{~W} / \mathbf{m}^{2} / \mathrm{K}$ \\
$k_{M}$ & 17.4 & $\mathrm{~W} / \mathbf{m} / \mathrm{K}$ \\
$k_{P}$ & 600.0 & $\mathrm{~W} / \mathbf{m} / \mathrm{K}$ \\
\hline
\end{tabular}

Table II

\begin{tabular}{ccccc}
\hline $\begin{array}{c}\text { Volume fraction } \\
\text { (size) }\end{array}$ & $k$ (exp.) & Nan et al. $^{\mathrm{a}}$ & Bruggeman $^{\mathrm{b}}$ & Richter and Viljoen \\
\hline $0.1(2 \mu \mathrm{m})$ & 1.05 & 1.08 & 1.07 & 1.06 \\
$0.2(2 \mu \mathrm{m})$ & 1.15 & 1.17 & 1.15 & 1.17 \\
$0.1(0.25 \mu \mathrm{m})$ & 0.70 & 0.90 & 0.90 & 0.75 \\
$0.2(0.25 \mu \mathrm{m})$ & 0.60 & 0.80 & 0.80 & 0.58 \\
$0.3(0.25 \mu \mathrm{m})$ & 0.50 & 0.72 & 0.70 & 0.49 \\
\hline
\end{tabular}


The thermal conductivity of composites of diamond and zinc sulfide has been measured for different volume fractions of diamond. Two sizes [radius] of diamond particles have been considered; $0.25 \mathrm{jim}$ and 2.0 $\mathrm{jim}$. The average size of the $\mathrm{ZnS}$ particles is $1-2 \mathrm{jim}$, according to Every at aL' ${ }^{\circ}$ Other data are given in Table I. The effective thermal conductivities are reported in Table II, scaled by the matrix value Our model compares quite well with the experimental results. When particles are sufficiently large, the Kapitza resistance effect diminishes to a point where it can be neglected. This is consistent with the results for $2.0 \mathrm{jim}$ particles. When the particles are small, the Kapitza resistance effect is enhanced, Our theory makes provision for resistance at diamond - diamond contacts. In most models homo boundaries are not considered, but especially in the case of diamond, it will be extremely difficult to remove all pores at the interface of two particles. The problem is to assign a value to the Kapitza resistance in this case. We have used a value of $h A$ $=3 \times 10 \mathrm{~m} 2 \mathrm{KIW}$, since it is expected that the Kapitza resistance would be much lower than at zinc diamond interfaces. The Kapitza resistance at $\mathrm{ZnS}-\mathrm{ZnS}$ contacts has been neglected. The model compates well with the other two models for the larger particle size. However, these models do not account for particle particle interaction and thus deviations can be expected at higher particle concentrations. The other models do not fare well with the smaller particle sizes. Possible explanations include the nonsphericity of the diamonds. Nan et aL12 actually showed that the model and experimental data could be better conciliated when ellipsoidal shapes are used.

This model holds definite advantages over existing models, the most important improvement is the consideration of statistics. Statistics feature in the proliferation of pathways as more layers are considered, each of diminishing contribution to the total current - this is reflected by the growth in terms of the Pascal triangle [cf. summations on the right hand side of Equation. [29]]. The probability of progression involves statistics of the packing. Even random packings can be treated if information on zenithal and azimuthal distribution of coordination are known. The model is also applicable over the range from dilute to highly concentrated loads of the particulate phase. The model correctly describes the dependence of the transport properties on film thickness at the extreme of very thin films. However, there are a few limitations of the model. In its current form the model does not account for particle shape. The model is limited to two species, so it is obvious that additional species will introduce considerable more complexity in the problem.

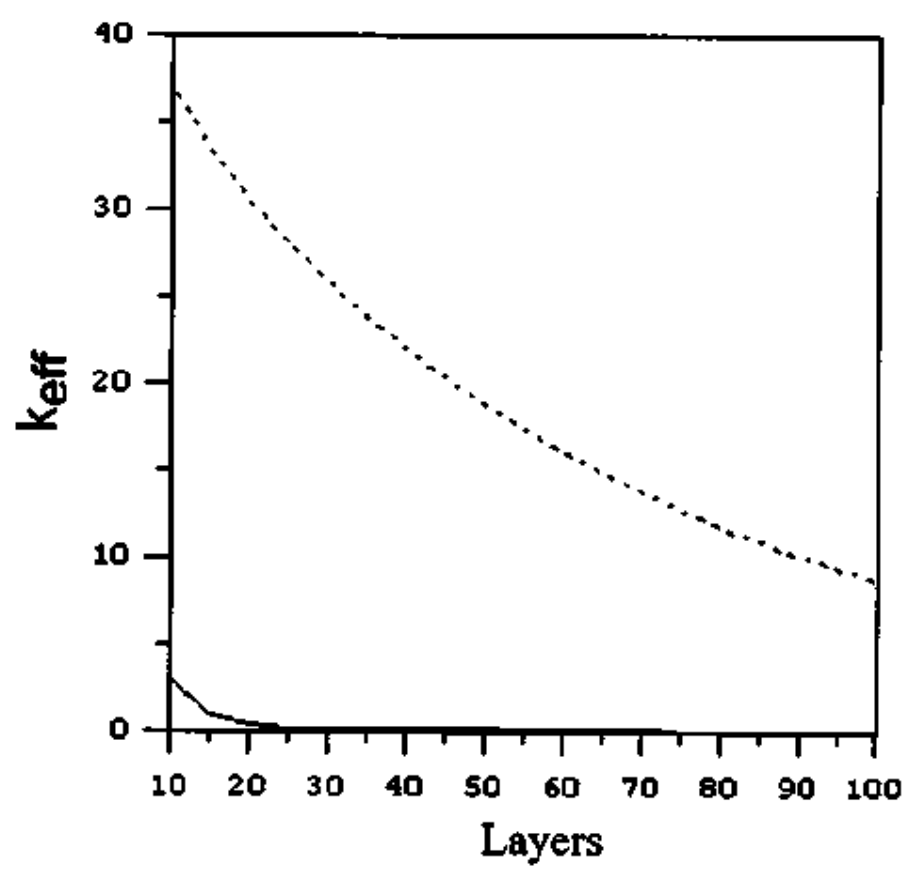

FIGURE 7. Effective thermal conductivity when one species is nonconducting. 
In the special case $k B=0$, Equation. [29] reduces to the very simple form

$$
k_{\text {eff }}=\rho(k+1)\left[\frac{P_{A}^{k}}{\left(1+k L_{A}\right) / k_{A}+k /\left(\Delta x h_{A}\right)}\right]
$$

It is not common practice to consider Kapitza resistances between particles of the same species. Although sound velocities vary along different principal directions of an anisotropic crystal, this effect is homogenized in randomly oriented particles. The reason for its consideration is the inclusion of in homogeneities at the interfaces.

Equationuation [30] strongly depends on the number of layers. In Fig. 7 the effective thermal conductivity is plotted as a function of the layer depth. Two concentrations of species A have been used. The dash line presents the effective thermal conductivity for $p=O . ? S$ and the full line presents $p=0.5 O$. The conductivity decreases dramatically in the more dilute case, due to the small probability to find a continuous A chain. The important point is that the effective conductivity becomes strongly dependent on the thickness of the medium and this result must be juxtaposed to the result shown in Fig.6.

\section{ACKNOWLEDGMENT}

C.R. and H.J.V. acknowledge the financial support of the National Science Foundation through Grant No. CTS0096381.

\section{APPENDIX}

The expression for $\mathrm{Fl}$ is written as

$$
\begin{aligned}
P_{2}^{d}= & P_{1}^{4}+P_{d}^{*}=P_{1}^{4}+4 \rho(1-\rho)^{5}\left[1-(1-\rho)^{3}\left\{1+3 \rho+3 \rho^{2}+\rho^{3}\right\}\right]+4 \rho^{2}(1-\rho)^{5}\left[1-(1-\rho)^{5}\left\{1+5 \rho+10 \rho^{2}+10 \rho^{3}+5 \rho^{4}\right.\right. \\
& \left.\left.+\rho^{5}\right\}\right]+2 \rho^{2}(1-\rho)^{5}\left[1-(1-\rho)^{6}\left\{1+3 \rho+3 \rho^{2}+\rho^{3}\right\}^{2}\right]+4 \rho^{3}(1-\rho)^{5}\left[1-(1-\rho)^{7}\left\{1+7 \rho+21 \rho^{2}+35 \rho^{3}+35 \rho^{4}\right.\right. \\
& \left.\left.+21 \rho^{5}+7 \rho^{6}+\rho^{7}\right\}\right]+\rho^{4}(1-\rho)^{5}\left[1-(1-\rho)^{8}\left\{1+8 \rho+28 \rho^{2}+56 \rho^{3}+70 \rho^{4}+56 \rho^{5}+28 \rho^{6}+8 \rho^{7}+\rho^{8}\right]\right\} .
\end{aligned}
$$




\section{References:}

J. C. Maxwell, A Treatise on Electricity and Magnetism., 3rd ed. [Oxford Universily Press, Oxford 1904].

R. Landauer, J. Appi. Phys, 23, 779 [1952].

E. H. Kerner, Proc. Phys. Soc. London, Sect. B 69, 802 [1956]. 4Z. Hashin, J. Compos. Mater 2, 284 [1968].

G. V Milton, The Theory of composites [Cambridge University Press, New York, 2002].

D. ter Haar, The Collected Papers of EL. Kapitza [Macmillan. New York, 1964].

Y. Benvenisle and T. Miloh, tnt. J. Eng. Sci. 24, 1537 [1986].

Y. Benveniste, 1. AppI. Phys. 61, 2840 [1987].

D. P. H. Hasselman and L. F. Johnson, 1. Compos. Mater. 21, 508 [1987].

A. G. Every, Y. Tzou, D. P. H. Hasselman, and R. Raj, Acta Metall. Mater 40, 123 [1992].

R. E. Meredith and C. W. Tobias, Adv. Electrochein. Electrochem, Eng. 2, 15 [1962].

C. W Nan et aL, J. Appi. Phys. 81, 6692 [1997],

C. Richter and H. 3. Viljoen, Thermochim. Acta 384, 315 [2002],

M. Kurashige, M. Mishima, and K. Imai, 3. Therm, Stresses 22, 713 [1999]. 